\title{
Modeling carcass removal time for avian mortality assessment in wind farms using survival analysis
}

\section{Regina Bispo, Joana Bernardino, Tiago A. Marques \& Dinis Pestana}

\section{Environmental and Ecological} Statistics

ISSN 1352-8505

Environ Ecol Stat

DOI 10.1007/s10651-012-0212-5

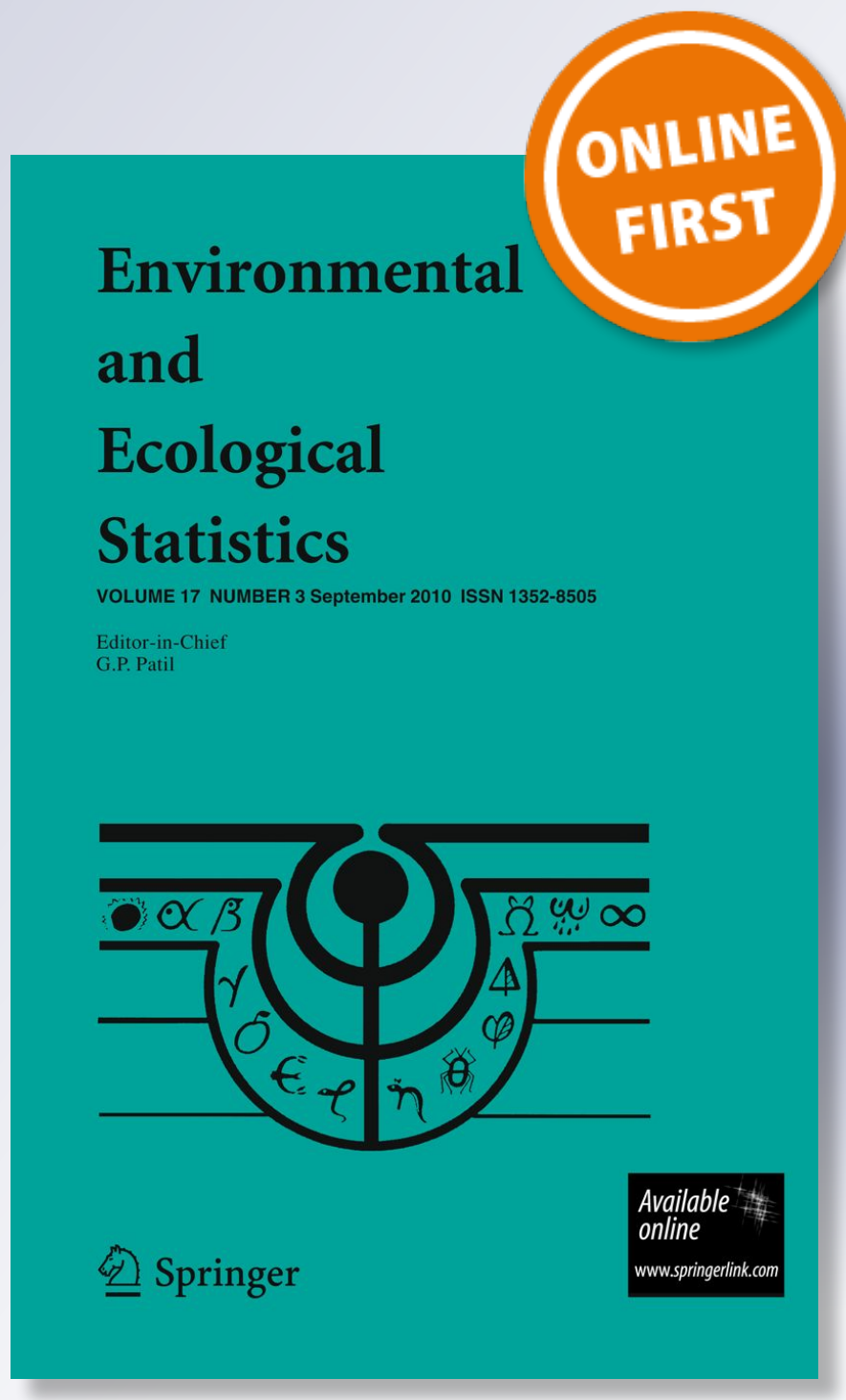

照 Springer 
Your article is protected by copyright and all rights are held exclusively by Springer Science+Business Media, LLC. This e-offprint is for personal use only and shall not be selfarchived in electronic repositories. If you wish to self-archive your work, please use the accepted author's version for posting to your own website or your institution's repository. You may further deposit the accepted author's version on a funder's repository at a funder's request, provided it is not made publicly available until 12 months after publication. 


\title{
Modeling carcass removal time for avian mortality assessment in wind farms using survival analysis
}

\author{
Regina Bispo • Joana Bernardino • \\ Tiago A. Marques • Dinis Pestana
}

Received: 19 January 2011 / Revised: 8 June 2012

(C) Springer Science+Business Media, LLC 2012

\begin{abstract}
In monitoring studies at wind farms, the estimation of bird and bat mortality caused by collision must take into account carcass removal by scavengers or decomposition. In this paper we propose the use of survival analysis techniques to model the time of carcass removal. The proposed method is applied to data collected in ten Portuguese wind farms. We present and compare results obtained from semiparametric and parametric models assuming four main competing lifetime distributions (exponential, Weibull, log-logistic and log-normal). Both homogeneous parametric models and accelerated failure time models were used. The fitted models enabled the estimation of the carcass persistence rates and the calculation of a scavenging
\end{abstract}

Electronic supplementary material The online version of this article (doi:10.1007/s10651-012-0212-5) contains supplementary material, which is available to authorized users.

R. Bispo ( $\square)$

ISPA-Instituto Universitário, Rua Jardim do Tabaco, 34, 1149-041 Lisbon, Portugal

e-mail: rbispo@ispa.pt

R. Bispo

Departamento de Estatística e Investigação Operacional, Faculdade de Ciências da Universidade de Lisboa, Bloco C6, Piso 4, Campo Grande, 1749-016 Lisbon, Portugal

\section{R. Bispo $\cdot$ T. A. Marques $\cdot$ D. Pestana}

CEAUL, Centro de Estatística e Aplicações da Universidade de Lisboa,

Bloco C6, Piso 4, Campo Grande, 1749-016 Lisbon, Portugal

J. Bernardino

Bio3, Estudos e Projectos em Biologia e Valorização de Recursos Naturais, Rua D. Francisco Xavier de Noronha, 37B, 2800-092 Almada, Portugal

T. A. Marques

Centre for Research into Ecological and Environmental Modeling, University of St. Andrews, The Observatory, St. Andrews, Scotland KY16 9LZ, UK 
correction factor for avian mortality estimation. Additionally, we discuss the impact that the distributional assumption can have on parameter estimation. The proposed methodology integrates the survival probability estimation problem with the analysis of covariate effects. Estimation is based on the most suitable model while simultaneously accounting for censored observations, diminishing scavenging rate estimation bias. Additionally, the method establishes a standardized statistical procedure for the analysis of carcass removal time in subsequent studies.

Keywords Accelerated failure time model - Bias correction factor · Lifetime distribution $\cdot$ Persistence rate $\cdot$ Survival analysis

\section{Introduction}

Bird and bat collision with man-made structures such as wind turbines, communication masts, power lines, buildings and fences is a well known phenomenon, extensively described in the literature (e.g. Barrios and Rodriguez 2004; Gelb and Delacretaz 2006; Hass et al. 2005; Newton 1998; Smallwood and Thelander 2004; Thelander and Smallwood 2007). Considered as a potential cause of significant avian mortality, collision is pointed out by some authors as the greatest unintended human cause of avian fatalities (e.g. Kelm et al. 2004). Currently, fatality of flying vertebrates through collision with rotating turbine rotor blades and other structures in wind farms receives most attention as consequence of the growing risk posed by the rapid increase in the number of wind turbines worldwide (Drewitt and Langston 2008).

Understanding the real impact of wind farm projects on avian and bat populations implies mortality estimation. To achieve that goal, appropriate monitoring has to be conducted whenever a wind farm project is consented.

To estimate real mortality, the observed mortality (number of carcasses found) needs to be adjusted for carcasses of animals which die but became unavailable for detection, either by scavenger removal, decomposition or any other cause. To quantify removal, wind farm monitoring plans include removal trials. Typically, in these trials a certain number of carcasses is randomly placed around the wind turbines for a a priori fixed period of time and time until removal is recorded.

Currently available methods to accommodate mortality estimates for removal vary greatly and in some cases are poorly described. Several authors (e.g. Kunz et al. 2007; Arnett et al. 2008) recently mentioned the lack of a reliable scavenging correction factor as an important source of unreliability in bird and bat mortality estimation. Next, we describe the currently applied methods used in practice to estimate scavenging rates. The described methodological heterogeneity illustrates the need for a unified estimation solution in order to ensure a correct evaluation of carcass removal and to contribute to find reliable and comparable mortality estimates between wind farms worldwide.

One of the most popular procedures estimates the scavenging removal by the mean length of time that a carcass remains on the study area before removal, based on the assumption that time until removal $(t)$ follows an exponential distribution with density $f(t)=\rho \exp (-\rho t)(t>0, \rho>0)$. Therefore, the mean length of time until removal 
is estimated by maximum likelihood by $\sum_{i=1}^{s} t_{i} /\left(s-s_{c}\right)$, where $t_{i}$ is the time since placement of carcass $i, s$ is the total number of carcasses planted for the trial and $s_{c}$ is the number of censored observations with constant value given by the time planned to end the experiment (e.g. Jonhson et al. 2003, 2004; Erickson et al. 2004).

Also assuming an exponential distribution for carcass persistence time, Huso (2010) adjusts mortality estimation for scavenging removal calculating the average probability of persistence of a carcass as $\hat{r}=(1 / I) \int_{x=0}^{I} \exp (-x / \bar{t}) d x$, where $I$ represents the length of the interval under consideration and the inverse of the mean persistence time $(\bar{t})$ estimates the $\rho$ parameter of the exponential distribution.

Barrios and Rodriguez (2004) estimated the number of birds carcasses that were removed by fitting an exponential function to the time of disappearance. These authors interpret this function as giving the rate of disappearance, and thus the removal probability, although the presented equations were neither probability density functions or probability distribution functions. They estimate the scavenging correction factor by averaging this rate of disappearance over the time elapsed between searches.

Other authors adjust observed mortality for scavenger removal by dividing the number of carcasses remaining at the end of a time period by the number of carcasses at the beginning of the period (e.g. Kerlinger 2002; Schmidt et al. 2003; Anderson et al. 2004; Brown and Hamilton 2006). Some authors (e.g. Schmidt et al. 2003; Brown and Hamilton 2006) calculate this proportion at the end of several weekly periods and adopt the correction factor as being the average of weekly values. Instead of finding the proportion of unscavenged carcasses at the end of a search cycle, Jain et al. (2007) calculated the proportion of removed carcasses after approximately half the actual search cycle, based on the assumption that the probability of a collision event is uniformly distributed between searches. Kerns et al. (2005) conducted trials for 21 days and estimated carcass persistence rate based on the empirical cumulative probability distribution by averaging the daily proportions of unscavenged carcasses for 7 days periods, $\frac{1}{7} \sum_{t=t_{0}}^{t_{1}}(1-P(T \leq t))$, with $\left(t_{0}, t_{1}\right)$ being $(1,7),(8,14)$ and $(15,21)$ days.

Linz et al. (1991) estimated carcass removal by scavengers using the product-limit life table method (Kaplan and Meier 1958). Based on Linz et al. (1991), Osborn et al. (2000) explicitly mention the use of nonparametric survival analysis methods (Kaplan-Meier) to analyze scavenging data at a wind farm site. These authors also estimate the percentage of unscavenged carcasses by dividing the number of carcasses remaining at the end of a time period by the number of carcasses at the beginning of the period. Rivera-Milan et al. (2004) and Kostecke et al. (2001) used survival analysis to estimate removal rates of bird carcasses in non wind farm related contexts. These authors used parametric survival analysis methods, modeling data through an exponential regression. Tobin and Dolbeer (1990), also working in a different theoretical context, estimated bird carcass persistence rates using an accelerated failure time model, by fitting a Weibull distribution to the time that carcasses remained in place until removal.

The data collected from carcass removal trials are classical examples of survival times. Their underlying distribution is typically positively skewed and often includes censored observations. The presence of censored observations is common when dealing with environmental data (Thompson and Nelson 2003) and its analysis has received 
attention in the literature (e.g. Akritas et al. 1994). This type of data is not amenable to standard statistical procedures and proper survival analysis should be used instead (Collett 2003).

Survival analysis includes nonparametric, semiparametric and fully parametric methodologies. Modeling survival data by using nonparametric procedures has the advantage to be unrestricted to a particular probability distribution resulting in a more flexible methodology, of wider applicability, but limits inference and prediction. A semiparametric approach allows to go further when the study involves the analysis of several variables as predictors of survival times. Because it does not assume any particular form of probability distribution for the survival times, in some cases, it is preferred to avoid errors related to the misspecification of the probabilistic model. Nevertheless, parametric methods, by assuming a specific form to the underlying data distribution, may allow more precise inferences (Collett 2003), although, in this case, estimates and their corresponding variances depend heavily on the validity on the assumptions. Efron (1977) and Oakes (1977) showed in particular that, under certain conditions, parametric survival models can lead to more efficient parameter estimates than the Cox model.

Using data sets from ten Portuguese wind farms we compare different survival analysis strategies (semiparametric and parametric) that can be used to model carcass removal time. We explore the impact that different modeling assumptions have on mortality estimates. Ultimately, we aim to establish a reliable statistical methodology for analyzing data from removal trials that avoids reporting findings exclusively on the grounds of empirical estimates promoting instead the use of adequate statistical models as a consequence of proper comparative goodness-of-fit analysis regarding diverse plausible models.

In the subsequent sections we describe carcass removal trials from which data was collected (Sect. 2), detail the statistical methods (Sect. 3), define the scavenging correction factor for mortality estimation (Sect. 4) and comment on the statistical findings of our research (Sect. 5). In the last section, we highlight the main conclusions, the practical value and the importance of the work developed.

\section{Carcass removal trials}

The motivating data comes from carcass removal trials conducted in ten wind farms located in the north and center of Portugal (for confidentiality reasons site are coded from WF1 to WF10). Trials were spread over two seasons (May/June and September/October or January/February and July/August) to assess the potential effects of weather conditions. Complete and fresh carcasses of parakeets (Melopsittacus undulates), quails (Coturnix coturnix) and partridges (Alectoris rufa) were used to represent three bird size classes - small (length under $15 \mathrm{~cm}$ ), medium (length between 15 and $25 \mathrm{~cm}$ ) and large (length above $25 \mathrm{~cm}$ ) — aiming to reflect the potential effect of carcass size in scavenging rates. Bird carcasses were obtained in avian breeding facilities. The carcasses were placed in the area of influence of the wind farm at a minimum distance of $500 \mathrm{~m}$ from each other. The number of carcasses placed in each trial varied between 20 and 80, according to the size of the farm. The carcass size class was chosen 
randomly. After their placement, all carcasses were monitored daily, every morning, recording time until removal for a maximum period of 20 days.

\section{Statistical analysis}

A failure time $(T)$ distribution is completely specified by its survivor function, $S(t)=$ $P(T>t)$. This function represents the probability that a subject survives from the time origin to some time beyond $t(t>0)$. In survival analysis, the hazard function, $h(t)=\lim _{\delta t \rightarrow 0}[P(t \leq T<t+\delta t \mid T \geq t) / \delta t]$, is also of interest because it expresses the risk of the event at time $t$.

Assuming that carcass removals occur independently of one another, the survivor function can be estimated nonparametrically using the Kaplan-Meier estimator (1958). This estimator, formed as a product of estimated probabilities, represents the empirical probability of surviving beyond time $t$. In the absence of censoring, the Kaplan-Meier estimate is simply the ratio between the number of carcasses not removed until time $t$ and the total number of carcasses placed in the beginning of the trial.

The semiparametric model known as the Cox regression model (1972) is a proportional hazards model in which the hazard at time $t$ is linked to the vector of $p$ covariates for the $i$-th subject $(i=1, \ldots, n), \mathbf{x}_{i}^{\prime}=\left(x_{1 i}, \ldots, x_{p i}\right)$ as $h_{i}(t)=h_{0}(t) \exp \left(\mathbf{x}_{i}^{\prime} \gamma\right)$, where $\gamma=\left(\gamma_{1}, \ldots, \gamma_{p}\right)^{\prime}$ is the vector of the unknown regression coefficients for the explanatory variables in the model and $h_{0}(t)$ represents the reference hazard for a subject with $\mathbf{x}=\mathbf{0}$. A positive/negative coefficient $\left(\gamma_{j}, j=1, \ldots, p\right)$ indicates that the hazard rate increases/decreases with the covariate value. In this model, covariates are assumed to act multiplicatively on the reference hazard and $\exp \left(\gamma_{j}\right)$ defines the relative hazard. Hence, the Cox regression model allows the interpretation of the results in terms of the relative risk.

Parametric survival analysis is based on a distributional assumption. There are several probability distributions described in the literature as suitable for survival data. The exponential, Weibull, log-logistic and log-normal distributions were used to model the removal process as they are among the most frequently used (e.g. Kalbfleisch and Prentice 2002; Lawless 2003).

The Weibull distribution with survivor function defined by $S(t)=\exp \left\{-(\rho t)^{\gamma}\right\}(\rho$, $\gamma>0$ ) has a monotonic decreasing hazard for a shape parameter less than one $(\gamma<1)$. Hence, it seemed a plausible model for scavenger removal trials, as the risk of removal is likely to be decreasing. However, when modeling carcass removal time it is possible to admit situations in which removal risk could first increase (because of smell, for instance) and then decrease. In these situations, the log-logistic and the lognormal models, whose hazard functions admit a positive mode, may be more adequate than the Weibull model. The corresponding survivor functions are given respectively by $S(t)=\left[1+(\rho t)^{\kappa}\right]^{-1}(\rho, \kappa>0)$ and $S(t)=1-\Phi[(\log t-\mu) / \sigma](\sigma>0)$ where $\Phi(\cdot)$ is the normal cumulative function. An a priori exponential distribution, for which $S(t)=\exp \{-\rho t\}$, seems implausible for scavenger removal times, because the implicit hazard of removal is constant. Nonetheless this distribution was included in our study because it is the most commonly used distribution in wind farm mortality estimation (e.g. Jonhson et al. 2003; Erickson et al. 2004; Fiedler 2004; Huso 2010). 
The previously mentioned probability models can be generalized into regression models to account for covariates effect. A general family of parametric survival regression models that encompasses all the above mentioned distributions is the accelerated failure time model (e.g. Kalbfleisch and Prentice 2002). These regression models can be represented in a general form in which the hazard at time $t$ for the $i$-th subject is given by $h_{i}(t)=h_{0}\left[t / \exp \left(\mathbf{x}_{i}^{\prime} \alpha\right)\right] \exp \left(-\mathbf{x}_{i}^{\prime} \alpha\right)$ where $\alpha=\left(\alpha_{1}, \ldots, \alpha_{p}\right)^{\prime}$ is the vector of the unknown regression coefficients of the $p$ covariates. The survivor function for the $i$-th individual is then given by $S_{i}(t)=S_{0}\left(t / \exp \left(\mathbf{x}_{i}^{\prime} \alpha\right)\right)$. In this model, explanatory variables act multiplicatively directly on the survival time (recall that in Cox regression model covariates are modeled as acting multiplicatively on the baseline hazard) and $\exp \left(\alpha_{j}\right)(j=1, \ldots, p)$ reflects the impact that a certain condition has on the baseline time scale. The quantity $\exp \left(-\alpha_{j}\right)$, termed acceleration factor, indicates the acceleration (if $>1$ ) or the deceleration (if $<1$ ) on the time of an event under a specific condition, relative to a reference situation. Cox and parametric survival regression models are likely to present coefficient estimates with opposite signs for the same covariates. This change in sign occurs because while the Cox model uses covariates to model the hazard rate, the parametric regressions use them to model directly the survival times. Hence, a positive sign of a coefficient in the parametric regression models indicates a deceleration on the event time, i.e., a hazard decrease. A negative sign represents the opposite.

Parametric accelerated failure time models can be unified by a log-linear formulation. This formulation may be used to give a useful general form for the survivor function. Considering the variable $T_{i}$ that represents the removal time for the $i$-th carcass, $\log T_{i}$ can be related to the vector of the covariates, $\mathbf{x}_{i}^{\prime}$, via $\log T_{i}=\mu+\mathbf{x}_{i}^{\prime} \alpha+\sigma \varepsilon_{i}$ where $\mu$ and $\sigma$ are, respectively, the intercept and the scale parameter and $\varepsilon_{i}$ is the error variable. The survivor functions for exponential, Weibull, log-logistic and log-normal regression models are then given respectively by

$$
\begin{aligned}
& S_{i}(t)=\exp \left[-\exp \left(\log t-\mu-\mathbf{x}_{i}^{\prime} \alpha\right)\right] \\
& S_{i}(t)=\exp \left[-\exp \left(\frac{\log t-\mu-\mathbf{x}_{i}^{\prime} \alpha}{\sigma}\right)\right] \\
& S_{i}(t)=\left[1+\exp \left(\frac{\log t-\mu-\mathbf{x}_{i}^{\prime} \alpha}{\sigma}\right)\right]^{-1} \\
& S_{i}(t)=1-\Phi\left[\frac{\log t-\mu-\mathbf{x}_{i}^{\prime} \alpha}{\sigma}\right]
\end{aligned}
$$

As, in this context, the final goal of inference is to use the fitted models to estimate carcass persistence probabilities, and these can depend heavily on the model selected, procedures that check model adequacy are particularly important. Both goodnessof-fit measures and plotting procedures were used to discriminate between the four mentioned competing parametric models (Bispo et al. in press). In this work we used the Cox-Snell residual plots to assess the overall fit of the models. The values for Akaike's Information Criterion (AIC) were used to compare the models' relative fit. In 
particular, AIC differences (defined, for model $l$, as $\triangle A I C_{l}=A I C_{l}-A I C_{\min }$, where $A I C_{\min }$ is the lowest AIC value for the fitted models) were determined for the fitted parametric models. As the best parametric distribution assumption is taken according to the lowest AIC value, AIC differences express the loss of information when the fitted model is used instead of the best adjusted model (Burnham and Anderson 2004).

The models efficiency for parameter estimates was compared using the Wald statistic, defined for a parameter $\beta$ by the ratio $\hat{\beta} / \hat{s e}(\hat{\beta})$ which converges in distribution to a standard normal distribution.

\section{Scavenging correction factor}

Assuming that the fatalities caused by collision occur uniformly over time, the scavenging correction factor $\left(r_{v k}\right)$ can be defined by

$$
r_{v k}=\frac{1}{I} \int_{0}^{I} S_{k}(t) d t
$$

where $I$ is the time interval between two consecutive searches and $S_{k}(t)$ is the parametric survivor function for the $k$-th condition. $r_{v k}$ expresses the average carcass persistence probability at the $v$-th search for the $k$-th condition defined by the covariates levels (or combination of levels).

Equation 5 generalizes the estimator proposed by Huso (2010). To reduce bias estimation, we propose the estimation of $S(t)$ based on the best fitted parametric survival model instead of assuming a priori the exponential distribution.

\section{Results}

In this study the removal time data was modeled accounting for season and carcass size effects. Farm site was not included as a covariate. Differences between inter-sites scavenging rates reported in previous work (Kerns et al. 2005) advise a site-specific based analysis as recently stressed by Arnett et al. (2008). Additionally, monitoring plans specific for each consented wind farm are often required by legislation, which implies site-specific trials and results.

Data were analyzed using $R$ Environment for Statistical Computing (R Development Core Team 2011). In particular, we used the "survival" package (Therneau and original Splus->R port by Thomas Lumley 2011).

\subsection{Comparative analysis of semiparametric and parametric modeling approaches}

Competing models were first analyzed regarding their goodness-of-fit. A graphical analysis of the Cox-Snell residuals (Fig. 1) shows that the models are generally well adjusted to all data sets, although some observations, corresponding to larger times until removal, are badly predicted by some less appropriate models, as shown by some 


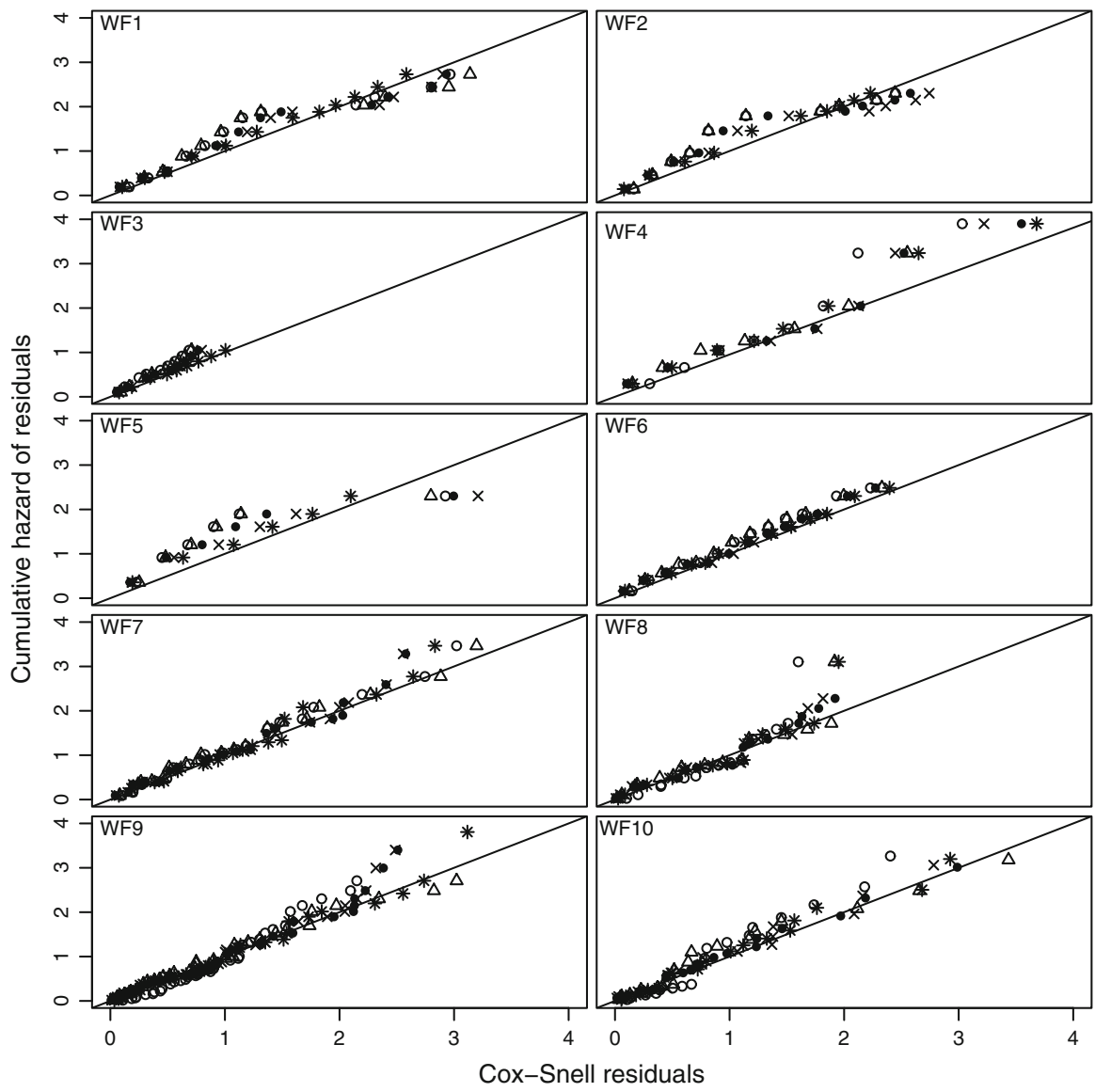

Fig. 1 Cox-Snell residuals for fitted models (circle exponential; triangle Weibull; times symbols Loglogistic; black circle Log-normal; asterisk Cox model)

extreme residual values which deviate from the expected linear relationship between the Cox-Snell residuals and the cumulative hazard of residuals for an appropriate model.

Table 1 presents $\triangle A I C$ values for the fitted parametric models. For WF1, WF3, WF4, WF6 and WF7, AIC differences indicate the log-normal model as the best fit. At WF2, WF5 and WF10, the log-logistic model presents the best fit. For the two remaining sites (WF8 and WF9) the Weibull based model presented the lowest AIC values. In some cases differences between AIC values regarding the different models were minimal (e.g. log-logistic and log-normal models for WF1), suggesting similar goodness-of-fit. As expected, the exponential was never the best model.

As the models' parameter estimates or their respective standard errors are not suitable for direct comparisons between models because the scale of the parameters differ, the Wald statistic was used to analyze comparatively the fitted models regarding their relative efficiency (Nardi and Schemper 2003). The obtained Wald statistic values 
Table 1 Differences between model's Akaike's information criterion and the minimum AIC

\begin{tabular}{lcrll}
\hline Wind farm & Exponential & Weibull & Log-logistic & Log-normal \\
\hline WF1 & 8.6 & 10.2 & 0.5 & 0.0 \\
WF2 & 18.9 & 20.9 & 0.0 & 1.4 \\
WF3 & 1.3 & 2.8 & 0.7 & 0.0 \\
WF4 & 18.1 & 7.9 & 4.7 & 0.0 \\
WF5 & 6.6 & 8.5 & 0.0 & 0.8 \\
WF6 & 9.2 & 10.6 & 1.8 & 0.0 \\
WF7 & 9.1 & 10.5 & 2.7 & 0.0 \\
WF8 & 10.6 & 0.0 & 3.6 & 3.0 \\
WF9 & 16.4 & 0.0 & 1.6 & 0.2 \\
WF10 & 5.2 & 0.7 & 0.0 & 0.1 \\
\hline
\end{tabular}

(Table 2) show that fitted models present different efficiency in parameter testing. For WF8, WF9 and WF10 the absolute values obtained under the best fitted parametric models (the Weibull model for WF8 and WF9 and the log-logistic model for WF10) were clearly higher than those obtained under the Cox model. There are other situations in which the gain in using a parametric model is only partial, as, e.g., at WF6, where the best parametric model (log-normal) leads to a higher efficiency in parameter testing when compared to the Cox model, only for the covariates representing carcass size. For some other data sets, as for WF1, both approaches present similar efficiency.

As the Wald statistic converges in distribution to a standard normal distribution it also gives information about covariates significance. In this study, the obtained values point to homogeneous removal times regarding season and carcass size factors in 6 (WF1 to WF6) out of the 10 wind farms (at WF5, the experimental design did not include body size effect). In WF7 and WF8, only season had a significant effect $(p<0.001)$ and in WF9 and WF10, both covariates seem important and have a significant effect on the removal times $(p<0.001)$.

\subsection{Models interpretation}

Both the empirical survivor functions (Kaplan-Meier) of carcass persistence and the best fitted parametric models (estimated survival functions in Online Resource 1) are illustrated in Figs. 2 and 3. Overall, plots in both figures show a very good agreement between the observed and the fitted survivor functions.

Figure 2 illustrates the removal processes for the six wind farms where available covariates where not found important to explain survival time. This figure shows that the rate of removal at WF4 and WF5 was the fastest, with only, respectively, 3 and $15 \%$ of the carcasses remaining after 7 days. In these two wind farms, complete removal occurred shortly after 13 and 15 days of placement, respectively. In the remaining four sites the removal process was slower and censored observations were registered. The slowest removal process was recorded at WF3. For this wind farm, the observed percentage of carcasses that remained unscavenged was never below $35 \%$. From 
Table 2 Wald statistic values for variables in models. Season and carcass size variables are represented in models using dummy variables

\begin{tabular}{|c|c|c|c|c|c|c|}
\hline Wind farm & Covariate & Cox & Exponential & Weibull & Log-logistic & Log-normal \\
\hline \multirow[t]{3}{*}{ WF1 } & Season 1 & -0.20 & 0.11 & 0.23 & -0.35 & -0.35 \\
\hline & Size 1 & -1.43 & 1.02 & 1.60 & 1.24 & 1.55 \\
\hline & Size 2 & -1.06 & 1.45 & 1.99 & 1.44 & 1.61 \\
\hline \multirow[t]{3}{*}{ WF2 } & Season 1 & -1.43 & 1.65 & 1.69 & 1.23 & 1.27 \\
\hline & Size 1 & $<0.01$ & -0.31 & -0.32 & 0.59 & 0.42 \\
\hline & Size 2 & 0.59 & -0.58 & -0.60 & -0.30 & -0.23 \\
\hline \multirow[t]{3}{*}{ WF3 } & Season 1 & 1.09 & -0.99 & -0.96 & -1.79 & -1.70 \\
\hline & Size 1 & -0.51 & 0.59 & 0.54 & 0.09 & 0.21 \\
\hline & Size 2 & -0.08 & 0.11 & 0.09 & -0.18 & -0.10 \\
\hline \multirow[t]{3}{*}{ WF4 } & Season 1 & -0.51 & 0.42 & 0.32 & 1.30 & 1.03 \\
\hline & Size 1 & 1.47 & -1.16 & -1.93 & -0.96 & -1.16 \\
\hline & Size 2 & -0.45 & 0.39 & 0.18 & 1.46 & 1.53 \\
\hline WF5 & Season 1 & -1.25 & 1.08 & 1.05 & 1.65 & 1.36 \\
\hline \multirow[t]{3}{*}{ WF6 } & Season 1 & -0.65 & 0.96 & 1.04 & 0.26 & 0.43 \\
\hline & Size 1 & -0.63 & 0.41 & 0.44 & 1.00 & 0.67 \\
\hline & Size 2 & -0.23 & 0.25 & 0.26 & 0.66 & 0.66 \\
\hline \multirow[t]{3}{*}{ WF7 } & Season 1 & 3.94 & -4.73 & -5.35 & -3.67 & -3.57 \\
\hline & Size 1 & 1.15 & -1.08 & -1.19 & -0.82 & -0.65 \\
\hline & Size 2 & -0.44 & 0.39 & 0.48 & 0.79 & 0.77 \\
\hline \multirow[t]{2}{*}{ WF8 } & Season 1 & -4.20 & 3.96 & 6.77 & 5.08 & 4.86 \\
\hline & Size 1 & -1.21 & 0.77 & 1.55 & 0.75 & 0.46 \\
\hline \multirow[t]{3}{*}{ WF9 } & Season 1 & -2.54 & 2.01 & 3.15 & 2.36 & 2.41 \\
\hline & Size 1 & 3.81 & -3.17 & -5.17 & -3.77 & -3.75 \\
\hline & Size 2 & 2.73 & -2.21 & -3.61 & -2.29 & -2.24 \\
\hline \multirow[t]{2}{*}{ WF10 } & Season 1 & 4.25 & -4.37 & -6.04 & -5.62 & -4.96 \\
\hline & Size 1 & -3.67 & 3.39 & 4.65 & 4.19 & 3.98 \\
\hline
\end{tabular}

The dichotomous variable Season is represented in the models using the dummy variable Season 1. The variable Size (carcass size) with three categories is represented in the models using the two dummy variables Size 1 and Size 2 (except for WF8 and WF10 where trials only included the medium and large carcasses sizes and hence variable Size is represented by one only dummy variable Size 1)

these plots, we see that the removal processes can be very distinct between sites, which reinforces the need for separate modeling.

In Fig. 3, the persistence decay for WF7 and WF8 is plotted by season. In these sites a similar scenario holds, in the sense that at both wind farms a rapid removal process was observed during the hot season and a slower process of removal prevailed during the cold season. However, at WF7, the removal rates were lower and censored times were observed in both seasons. The seasonal effect was more notorious at WF8, where 5 days since placement were enough to observe complete removal of avian carcasses during spring. During fall, complete removal was observed after 15 days and no censored observations were recorded. 


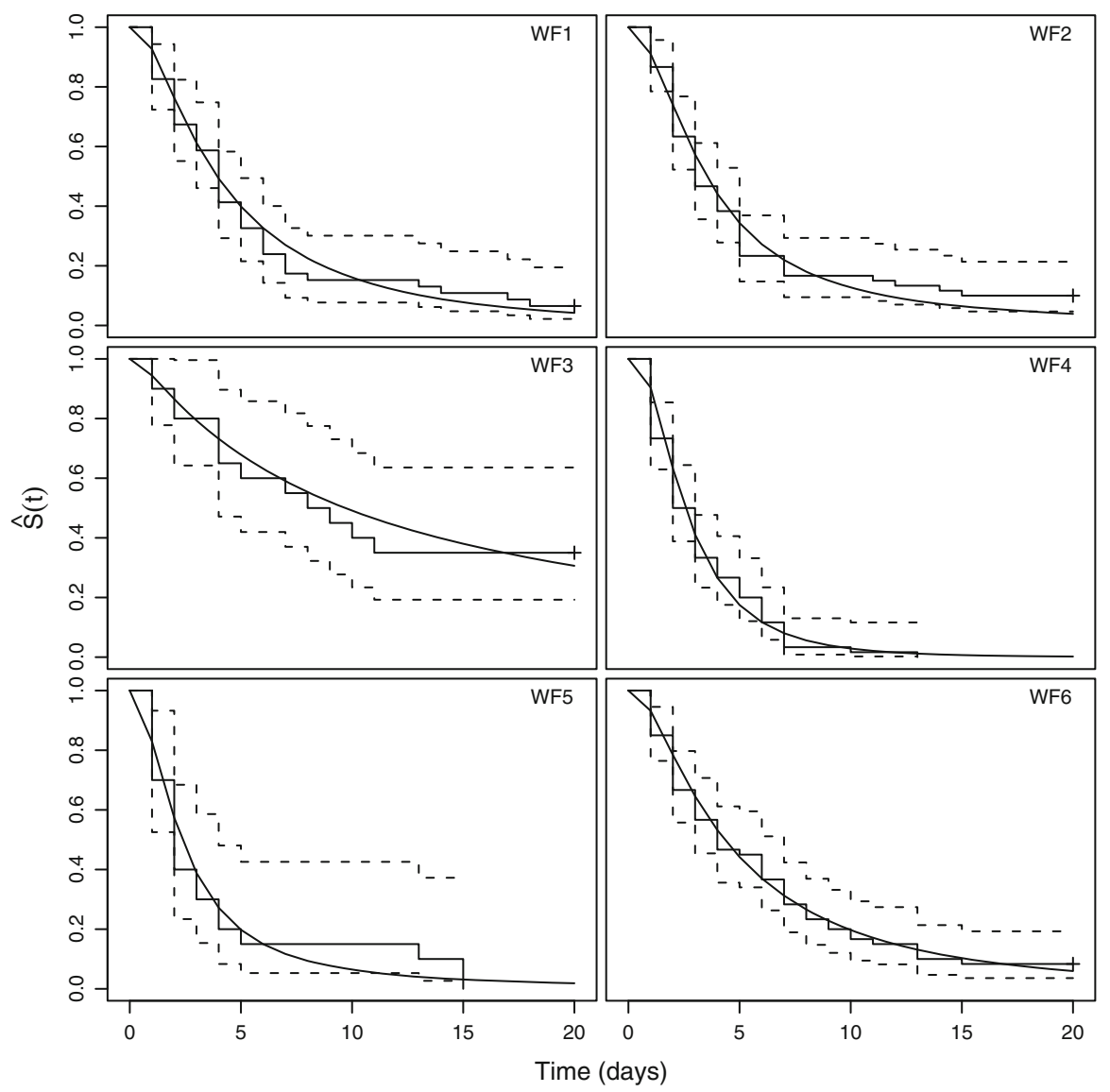

Fig. 2 Empirical survivor function (step functions) for homogeneous populations. Superimposed on the step functions are the survivor functions of the best fitted models. Plots represent estimated functions at WF1, WF2, WF3, WF4, WF5 and WF6 wind farms. The character (+) at the end of the step functions indicates the existence of right censored observations. Dashed lines represent the confidence interval bands for the empirical survivor functions

Plots regarding sites where both covariates significantly affected time until removal (WF9 and WF10) show a tendency towards higher persistence rates during the colder seasons depending, however, on the size of the carcass. In fact, at WF9, during the May/Jun trial, carcasses of medium and large sizes were completely removed by the fourth and sixth days, respectively, and small size carcasses persisted in place until nine days of placement. By contrast, during Sep/Oct, carcasses of medium and large sizes were completely removed after 7 and 6 days, respectively, and small size carcasses still remained unremoved after the end of the trial, originating censored observations. At WF10, a similar scenario was found. For the trial conducted in Jul/Aug, carcasses of medium size were completely removed shortly after 3 days of placement and the large size carcasses remained unscavenged until 15 days of placement, when complete removal occurred. During the Jan/Feb trial, medium size carcasses were completely 


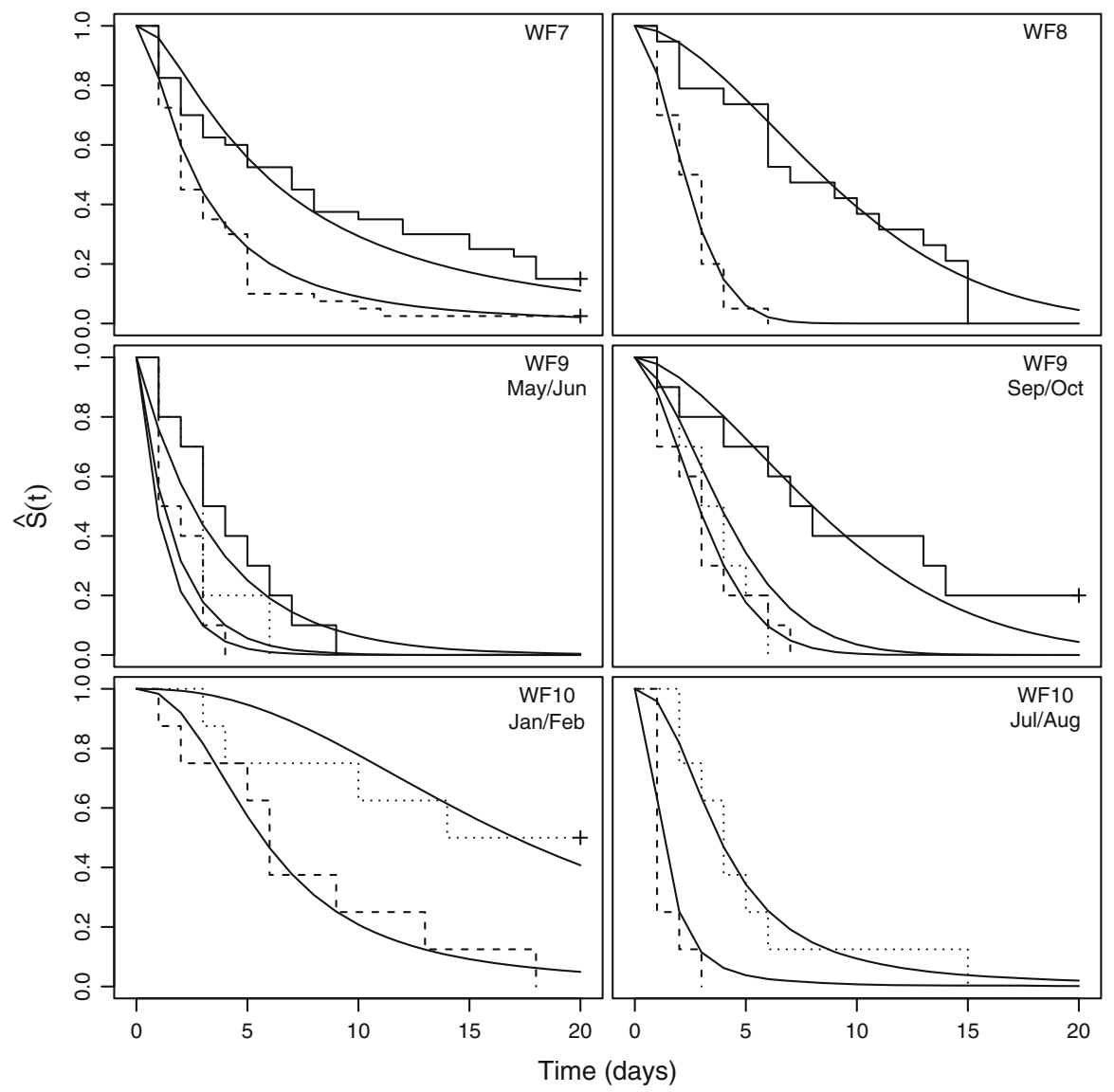

Fig. 3 Empirical survivor function (step functions) for nonhomogeneous populations. Superimposed on the step functions are the survivor functions of the best fitted models. Plots represent estimated functions at WF7 (solid line: Jan/Feb and dashed line: Jul/Aug), WF8 (solid line: Sep/Oct and dashed line: May/Jun), WF9 (solid lines: small size carcasses, dashed lines: medium size carcasses and dotted lines: large size carcasses) and WF10 (dashed lines: medium size carcasses and dotted lines: large size carcasses) wind farms. The character $(+)$ at the end of the step functions indicates the existence of right censored observations. Confidence interval bands for the empirical survivor functions were omitted for clarity

removed after 18 days of placement and large size carcass observations were censored at the end of the trial.

One of the advantages of the parametric approach is that, for non-homogeneous populations, it provides direct assessment of the fitted model in terms of the speed of event of interest. Hence, in this study, the acceleration factor $\left(\exp \left(-\alpha_{j}\right)\right.$, see Sect. 3), expresses the effect that a specific covariate has on removal speed (for detailed information about coefficients values see estimated survival functions in Online Resource 1).

At WF7, where the best model is the log-normal model, the acceleration factor value indicates a carcass removal process about $2(\exp (0.808)=2.24)$ times faster during Jul/Aug than during Jan/Feb. At WF8, where the best model is the Weibull 
model, the time of removal of a avian carcass during Sep/Oct was found to be about 4 times lower than that recorded during May/Jun.

The values of the acceleration factors show that, at WF9, the removal process of avian carcasses occurred following the decreasing order (acceleration factors in brackets): medium size-May/Jun (2.80), large-May/Jun (2.08), medium-Sep/Oct (1.67), large-Sep/Oct (1.24), small-May/Jun (1.00) and small-Sep/Oct (0.60). Hence, it seems that the carcass size effect can be stronger than the season effect.

At WF10 the removal followed the decreasing order (acceleration factors in brackets): medium-Jul/Aug (4.50), large-Jul/Aug (1.30), medium-Jan/Feb (1.00) and large-Jan/Feb (0.33). At this wind farm, the removal process was clearly faster for carcasses of medium size during Jul/Aug, with an almost five times faster removal than carcasses of medium size during Jan/Feb.

\subsection{Scavenging correction factor estimates}

In practice, the time period between two consecutive searches for carcasses in wind farms for which is necessary to calculate the scavenging correction factor can vary greatly. In this study, for illustration purposes, we calculated the average values for a time interval of 7 days, with no loss of generality.

Table 3 summarizes the weekly scavenging correction factors estimated from the best parametric fitted models for each studied site. The scavenging correction factor values clearly differ between the six farms where no covariates were found to be significant (WF1 to WF6). At WF4 and WF5, where the removal process was faster, only about $42-44 \%$ of the carcasses remained, on average, unscavenged during a period of time of 7 days. By contrast, at WF3, for the same time interval, $78 \%$ of the carcasses persisted, on average, due to the slow process of removal at this site.

The heterogeneity between WF7 and WF8 was even higher than the recorded for homogeneous populations. At WF8, the average rates show a more evident seasonal effect than the one found in WF7, as expected from the graphical representation of the survivor functions (Fig. 3).

In WF9 and WF10, the smallest correction factors were found for medium size carcasses. At WF9, the large size carcasses were removed after medium size carcasses and small size carcasses were the last to be removed. Within each carcass size category, removal process was always faster during summer than during fall. At WF10, the correction factors were much higher for larger size carcasses than for medium size carcasses.

5.4 Impact of the removal time model distributional assumption on the estimation of mortality

To emphasize the importance of considering different plausible models, we compare the scavenging correction factor estimates (complete set of values available in Online Resource 2) and evaluate the impact that the distribution assumption can have on the estimated mortality rate. 
Table 3 Estimated scavenging correction factors for a time interval of 7 days at each study site

\begin{tabular}{|c|c|c|c|c|}
\hline Wind farm & Distribution & Condition & & $\hat{r}$ \\
\hline WF1 & Log-normal & & & 0.58 \\
\hline WF2 & Log-logistic & & & 0.56 \\
\hline WF3 & Log-normal & & & 0.78 \\
\hline WF4 & Log-normal & & & 0.44 \\
\hline WF5 & Log-logistic & & & 0.42 \\
\hline WF6 & Log-normal & & & 0.62 \\
\hline \multirow[t]{2}{*}{ WF7 } & \multirow[t]{2}{*}{ Log-normal } & $\mathrm{Jan} / \mathrm{Feb}$ & & 0.71 \\
\hline & & Jul/Aug & & 0.46 \\
\hline \multirow[t]{2}{*}{ WF8 } & \multirow[t]{2}{*}{ Weibull } & May/Jun & & 0.35 \\
\hline & & Sep/Oct & & 0.84 \\
\hline \multirow[t]{6}{*}{ WF9 } & \multirow[t]{6}{*}{ Weibull } & May/Jun & Small & 0.65 \\
\hline & & & Medium & 0.27 \\
\hline & & & Large & 0.36 \\
\hline & & Sep/Oct & Small & 0.82 \\
\hline & & & Medium & 0.45 \\
\hline & & & Large & 0.57 \\
\hline \multirow[t]{4}{*}{ WF10 } & \multirow[t]{4}{*}{ Log-logistic } & $\mathrm{Jan} / \mathrm{Feb}$ & Medium & 0.74 \\
\hline & & & Large & 0.96 \\
\hline & & Jul/Aug & Medium & 0.23 \\
\hline & & & Large & 0.58 \\
\hline
\end{tabular}

If at some sites the estimates of the correction factor were quite homogeneous across the different competing models (e.g., WF1 and WF6), there are others sites (such as WF5 or WF9), in which the correction factor value clearly depends on the model distributional assumption, suggesting that the use of an incorrect model can severely bias mortality estimation.

Consider a carcass search protocol where weekly spaced searches are used. Further, consider that mortality rate (number of corpses per period of time) at the $v$-th search for the $k$-condition $\left(m_{v k}\right)$, can be simply estimated by (Jain et al. 2007)

$$
m_{v k}=\sum_{v=1}^{n} o_{v k} /\left(d \times r_{v k}\right)
$$

where $o_{v k}$ is the number of found carcasses, $r_{v k}$ is the scavenging correction factor (see Eq. 5). Suppose $d$, the detection probability is fixed at 0.25.

Assume that WF1 is being monitored and that after a period of 1 week, two bird carcasses per turbine were found. In this wind farm, estimated correction removal factors for a time interval of 7 days are $0.59,0.61,0.58$ and 0.58 , under the exponential, Weibull, log-logistic and log-normal regression models, respectively. Then, in this case, the estimated mortality (Eq. 6) would be, respectively, 14 (i.e., $2 /(0.25 \times 0.59)$ ), 
13,14 and 14 birds. In this case, differences in mortality estimates, due to the different distributional assumption of the removal process, are minor.

A similar comparison reveals that in WF5 adopting an exponential model instead of the best fitted log-logistic model, the mortality will be underestimated by $16 \%$ which turns out to be 624 birds in a year, considering the four turbines in this site.

Lastly, suppose that WF9 is being monitored and that a weekly average of five large size carcasses per turbine was found during Sep/Oct. In this case, the estimated mortality would be 40 and 35 birds under the exponential and the Weibull models, respectively. Hence, if working with the exponential rather than the best parametric model, mortality would be overestimated by five birds per week per turbine, i.e., 70 birds considering the 14 turbines in WF9. Similar computations lead to an underestimation of $(5 \times 14) \times(1 /(0.25 \times 0.36)-1 /(0.25 \times 0.55)) \approx 269$ birds per week during May/June resulting in a average net underestimation of $269-70=199$ birds per week during the 4 months May/June and Sep/Oct.

These examples illustrate the impact that model choice can have on the estimation of the parameters of interest.

\section{Discussion}

Previous studies estimate scavenger persistence rates either (1) empirically (e.g. Kerlinger 2002; Anderson et al. 2004; Kerns et al. 2005; Jain et al. 2007), which limits and may compromise inference; (2) based on a priori distribution (exponential distribution) (e.g. Jonhson et al. 2003; Erickson et al. 2004; Fiedler 2004; Huso 2010), or (3) using estimators not accounting for censored observations (e.g. Kerlinger 2002; Schmidt et al. 2003; Brown and Hamilton 2006).

Here, we propose a method for the analysis of scavenger removal data aiming to standardize procedures and overcome the above mentioned limitations. We compared a semiparametric with a fully parametric approach. Overall we found advantages in using the parametric methodology. This method enables, simultaneously, to model carcass removal times accounting for covariate effects and to estimate persistence probabilities for all covariate levels. With this methodology, estimation is based on the most suitable probability distribution and accounts for censored observations, diminishing bias. Also, the integration of the estimation problem with the analysis of covariates effects, avoids performing several univariate analysis controlling the overall probability of making any incorrect decision, regarding covariates effect. Based on this approach we formally define a scavenging correction factor (Eq. 5) applicable to any time interval between searches used in carcass search protocols in wind farms monitoring projects or any other projects that imply carcass removal evaluation (as, e.g, roads and power lines monitoring projects).

In this study, the proposed methodology was used to model ten real data sets which give a broad realistic idea about the application conditions and the usefulness of the method. In six out of the ten wind farms no significant effects of body size and/or season were found, but in the remaining wind farms covariates were found to be highly significant, substantially affecting removal process and persistence rates. This stresses the need for accounting these covariates in scavenger removal trials, but also 
motivates the study of other explanatory variables that might influence estimation (e.g., type of vegetation cover).

Typically, persistence rates were found to be higher during colder seasons and lower for hotter seasons. Medium and large carcasses were removed prior to small carcasses, regardless of the season. Thus, we concur with other authors (e.g. Morrison 2002) indications that a separate trials by season and size carcass design is needed when measuring scavenging removal bias. The scavenging correction factors clearly differed between wind farms, showing that adjusting mortality based on scavenging rates determined elsewhere is not useful, and that, instead, the time of removal must be determined within each site as recommended previously by other authors (e.g.Arnett et al. 2008).

In most wind farms, the log-normal and the log-logistic regression models fitted the removal time data better. In two study sites, the Weibull model adjusted better. The exponential model was never the best option. To model the time of removal we chose these four models, but others are available out there which might outperform these in practice. The recent study by Silva et al. (2010) characterizes the generalized exponential geometric distribution (GEG distribution), with cumulative distribution function $F(x ; \beta, p, \alpha)=((1-\exp \{-\beta x\}) /(1-p \exp \{-\beta x\}))^{\alpha},(x, \beta, \alpha>0, p \in$ $(0,1))$, covering in a single family a broad class of hazard functions (decreasing, increasing or a upside-down failure rate), depending on its parameters. So, if proven to provide a better fit, this model can represent an attractive alternative approach and be used to model removal trials data. To illustrate its use we present here an application of the GEG distribution to the WF4 and WF5 data trials. These data sets were chosen as they do not include censored observations and covariates were not statistically significant. The maximum likelihood estimates determined by fitting the GEG distribution to the mentioned data sets are $\hat{\alpha}=48.672, \hat{\beta}=0.370, \hat{p}=0.978$, for the WF4 data set and $\hat{\alpha}=33.908, \hat{\beta}=0.159, \hat{p}=0.990$, for the WF5 data set. Comparing these models with the previously fitted survival models, we found that the fit of the GEG model for the WF4 data set is in fact superior to the fit when using the log-normal model. At this point, however, there are no tools available to fit the GEG model in the presence of censored observations, which limits its current applicability in practice.

The parametrically estimated persistence rates clearly depended on the model distributional assumption. If, in some wind farms, estimated removal correction factors differed only slightly depending on the model (as, e.g., in WF1), other estimates differed substantially (as, e.g., in WF9). Hence, the study of different plausible models regarding its adequacy is crucial to reduce bias estimation of scavenging rates and increase confidence in inference results.

Acknowledgments This research was conducted in collaboration with BIO3-Estudos e Projectos em Biologia e Valorização de Recursos Naturais. The study was partially sponsored by national funds through the Fundação Nacional para a Ciência e Tecnologia, Portugal-FCT under the project PEstOE/MAT/UI0006/2011. Comments and suggestions made by the referees have been of the utmost importance in the preparation of the final version of this paper. 


\section{References}

Akritas MG, Ruscitti TF, Patil GP (1994) Statistical analysis of censored environmental data. In: Patil GP, Rao CR (eds) Handbook of statistics 12, Environmental Statistics. North-Holland, New York, pp 221-242

Anderson R, Neumann N, Tom J, Erickson W, Strickland MD, Bourassa M, Bay K, Sernka K (2004) Avian monitoring and risk assessment at the Tehachapi Pass Wind Resource Area. Tech. rep., National Renewable Energy Laboratory, Golden, Colorado, USA

Arnett E, Brown W, Erickson W, Fiedler J, Hamilton B, Henry T, Jain A, Johnson G, Kerns J, Koford R, Nicholson C, O'Connell T, Piorkowski M, Tankersley R (2008) Patterns of bat fatalities at wind energy facilities in North America. J Wildl Manag 72:61-78

Barrios L, Rodriguez A (2004) Behavioural and environmental correlates of soaring-bird mortality at onshore wind turbines. J Appl Ecol 41:72-81

Bispo R, Bernardino J, Marques TA, Pestana D (in press) Discrimination between parametric survival models for removal times of bird carcasses in scavenger removal trials at wind turbines sites. In: Studies in Theoretical and Applied Statistics. Springer, New York

Brown WK, Hamilton BL (2006) Monitoring of bird and bat collisions with wind turbines at the Summerview Wind Power Project, Alberta, 2005-2006. Report for Vision Quest Windelectric. In: Terrestrial and Aquatic Environmental Managers Ltd., Calgary, Alberta, Canada

Burnham KP, Anderson DR (2004) Understanding AIC and BIC in model selection. Sociol Methods Res 33:261-304

Collett D (2003) Modelling survival data in medical research. Chapman and Hall, Boca Raton, FL

Cox DR (1972) Regression models and life-tables. J R Statist Soc B 34:187-220

Drewitt A, Langston R (2008) Collision effects of wind-power generators and others obstacles on birds. Ann NY Acad Sci 1134:233-266

Efron B (1977) The efficiency of Cox's likelihood function for censored data. J Am Stat Assoc 72:557-565

Erickson WP, Jeffrey J, Kronner, Bay K (2004) Stateline wind project wildlife monitoring final report, July 2001-December 2003. Technical report peer-reviewed by and submitted to FPL Energy, the Oregon Energy Facility Siting Council, and the Stateline Technical Advisory Committee, Western EcoSystems Technology, Inc. and Northwest Wildlife Consultants, Inc

Fiedler JK (2004) Assessement of bat mortality and activity at Buffalo Mountain Windfarm, Eastern Tennessee. Master's thesis, The University of Tennessee. Available electronically at http://www. wind-watch.org

Gelb Y, Delacretaz N (2006) Avian window strike at an urban office building. The Kingbird 56:190-198

Hass D, Nipkow M, Fielder G, Schneider R, Haas W, Schürenberg B (2005) Protecting birds from powerlines. In: Nature and environment, vol 140. Council of Europe Publishing

Huso MMP (2010) An estimator of wildlife fatality from observed carcasses. Environmetrics 22:318-329

Jain A, Kerlinger R Pand Curry, Slobodnik L (2007) Annual report for the Maple Ridge Wind Power Project: postconstruction bird and bat fatality study-2006. Tech. rep., Curry and Kerlinger, LLC. Available electronically at http://www.wind-watch.org

Johnson G, Perlik M, Erickson W, Strickland MD (2004) Bat activity, composition, and collision mortality at a large wind plant in Minnesota. Wildl Soc Bull 32:1278-1288

Jonhson G, Erickson W, White J, McKinney R (2003) Avian and bat mortality during the first year of operation at the Klondike phase I wind project, Sherman County, Oregon. Tech. rep., WEST, Inc., Cheyenne. Available electronically at http://www.west-inc.com

Kalbfleisch JD, Prentice RL (2002) The statistical analysis of failure time data. Wiley, New York

Kaplan EL, Meier P (1958) Nonparametric estimation from incomplete observations. J Am Stat Assoc 53:457-481

Kelm D, Keck DC, Marty KL, Ball AJM, Niciu EE, Plat CT (2004) Effects of window angling, feeder placement and scavengers on avian mortality at Plate Glass. Wilson Bull 116(1):69-73

Kerlinger P (2002) An assessment of the impacts of Green Mountain Power Corporation's wind power facility on breeding and migrating birds in Searsburg, Vermont. Tech. rep., National Renewable Energy Laboratory, Golden, Colorado, USA. Available electronically at http://www.osti.gov/bridge

Kerns J, Erickson W, Arnett EB (2005) Bat and bird fatality at wind energy facilities in Pennsylvania and West Virginia. In: Arnett EB (ed) Relationships between bats and wind turbines in Pennsylvania and West Virginia: an assessment of bat fatality search protocols, patterns of fatality, and behavioral interactions with wind turbines. Bat Conservation International, Austin, Texas, USA, pp 24-95 
Kostecke RM, Linz GM, Bleier WJ (2001) Survival of avian carcasses and photographic evidence of predators and scavengers. J Field Ornithol 72:439-447

Kunz T, Arnett E, Cooper B, Erickson W, Larkin R, Mabee T, Morrison M, Strickland M, Szewczac J (2007) Assessing impacts of wind-energy development on nocturnally active birds and bats: a guidance document. J Wildl Manag 71:2449-2486

Lawless JF (2003) Statistical models and methods for lifetime data. Wiley, New York

Linz GM, Davis JE, Engeman RM, Otis DL, Avery ML (1991) Estimating survival of birds carcasses in cattail marshes. Wildl Soc Bull 19:195-199

Morrison M (2002) Searcher bias and scavenging rates in bird/wind energy studies. Tech. rep., National Renewable Energy Laboratory, Golden, Colorado, USA

Nardi A, Schemper M (2003) Comparing Cox and parametric models in clinical trials. Statist Med 22:35973610

Newton I (1998) Population limitation in birds. Ibis 149:453-467

Oakes D (1977) The asymptotic information in censored survival data. Biometrika 64:441-448

Osborn RG, Higgins K, Usgaard RE, Dieter CD, Neiger RD (2000) Bird mortality associated with wind turbines at the Buffalo Ridge Wind Resource Area, Minnesota. Am Midl Nat 143:41-52

R Development Core Team (2011) R: A language and environment for statistical computing. URL http:// www.R-project.org, ISBN 3-900051-07-0

Rivera-Milan FF, Zaccagnini ME, Canavelli SB (2004) Field trials of line transect surveys of bird carcasses in agro-ecosystems of Argentina's Pampas region. Wildl Soc Bull 32:1219-1228

Schmidt E, Piaggio A, Bock C, Armstrong D (2003) National wind technology center site environmental assessment: Bird and bat use and fatalities final report. Tech. rep., University of Colorado Boulder, Golden, Colorado, USA. Available electronically at http://www.doe.gov/bridge

Silva RB, Barreto-Souza W, Cordeiro GM (2010) A new distribution with decreasing, increasing and upside-down bathtub failure rate. Comput Stat Data Anal 54:935-944

Smallwood KS, Thelander C (2004) Developing methods to reduce bird mortality in the Altamont Pass Wind Resource Area. Final report by bioresource consultants to the California energy commission, California Energy Commission, Public Interest Energy Research, Environmental Area. Available electronically at http://www.energy.ca.go

Thelander C, Smallwood KS (2007) The Altamont pass wind resource area's effects on birds: a case history. In: de Lucas M, Janss GFE, Ferrer M (eds) Birds and wind farms: risk assessment and mitigation. Quercus, Madrid, pp 25-46

Therneau T, original Splus->R port by Thomas Lumley (2011) survival: Survival analysis, including penalised likelihood. URL http://CRAN.R-project.org/package=survival, R package version 2.36-5

Thompson ML, Nelson KP (2003) Linear regression with type I interval- and left-censored response data. Environ Ecol Stat 10:221-230

Tobin ME, Dolbeer RA (1990) Disapearance and recoverability of songbirds carcasses in fruit orchards. J Field Ornithol 61:237-242

\section{Author Biographies}

Regina Bispo received a M.Sc. degree in probability and statistics from Universidade de Lisboa and got her $\mathrm{PhD}$ at Universidade Técnica de Lisboa. She is an Assistant Professor at ISPA-Instituto Universitário and an invited Assistant Professor at Faculdade de Ciências da Universidade de Lisboa. She is also a member of CEAUL, Centro de Estatística e Aplicações da Universidade de Lisboa with interests in biostatistics and in particular in ecological statistics.

Joana Bernardino is a wildlife biologist in Bio3, occupying the position of project manager since 2007. She has been responsible for several biodiversity monitoring programs, mainly related to impact assessment of renewable energies projects on vertebrates. In 2008 she completed her master's degree in Ecology and Environmental Management at Faculdade de Ciências da Universidade de Lisboa.

Tiago A. Marques is a Research Fellow at the University of St Andrews, from where he obtained his $\mathrm{PhD}$ in statistics in 2007. TAM first degree was in Biology (Universidade of Lisboa 1998). He is a member of CEAUL, Centro de Estatística e Aplicações da Universidade de Lisboa. His main research areas are statistical methods applied to estimating animal abundance, in particular the use and development of distance sampling 
methods. More recently, he has been working on developing methods to estimate animal abundance from passive acoustic data.

Dinis Pestana got his PhD in Sheffield, UK (1974). Formerly Professor of Probability and of Biostatistics at the University of Lisbon, he is now research director at the CEAUL, Centro de Estatística e Aplicações da Universidade de Lisboa. 